\title{
Role of teledentistry in paediatric dentistry
}

\author{
Christopher K. Wallace, ${ }^{\star 1}$ Charlotte E. Schofield, ${ }^{1}$ Lucy A. L. Burbridge ${ }^{1}$ and Katherine L. O'Donnell ${ }^{1}$
}

\section{Key points}

Teledentistry has numerous applications within paediatric dentistry, both within and beyond the current COVID-19 pandemic.
Teledentistry reduced the need for face-to-face new patient appointments by one-third at Newcastle Dental Hospital.
Teledentistry may improve patient journeys and reduce inconvenience; however, quality of telephone consultation is dependent on initial referral quality.

\begin{abstract}
Introduction As a result of the COVID-19 pandemic, teledentistry has been used more frequently due to social distancing regulations to minimise the need for face-to-face attendances.
\end{abstract}

Aims Evaluate uses of teledentistry within Newcastle Dental Hospital's Paediatric Dentistry New Patient Service. Establishes potential roles of teledentistry within paediatric dentistry and advantages and disadvantages of this service method.

Methods Service evaluation of 653 new patient teledentistry appointments at Newcastle Dental Hospital across a onemonth period (13 May- 12 June 2020).

Discussion By implementing a telephone consultation as the first point of contact with our team, we were able to reduce the number of face-to-face appointments required by over a third. Teledentistry can be used for numerous applications within paediatric dentistry including initial triage, remote assessment, reinforcement of prevention, implementing initial management and building rapport.

Conclusion Teledentistry is an emerging field and has the potential to improve patient journeys, reduce waiting lists and reduce unnecessary face-to-face attendances which is especially important both within and beyond the current COVID-19 pandemic to maximise safety and minimise inconvenience for both parent and child. However, care must be taken to ensure a clinician is confident in their diagnosis prior to discharging or delaying management of a patient.

\section{Introduction}

Teledentistry is defined as the 'provision of real time and offline dental care such as diagnosis, treatment planning, consulting and follow-up via electronic transmission from different sites.' ${ }^{\text {T }}$ Teledentistry can take place in multiple formats; these are 'store and forward', remote monitoring and real-time video conferencing. 'Store and forward' is where a dental professional gathers information about a patient and forwards this on to a

'Paediatric Dentistry, The Newcastle upon Tyne NHS Foundation Trust, Newcastle Dental Hospital, Richardson Road, Newcastle upon Tyne, Tyne and Wear, NE2 4AZ, UK. *Correspondence to: Christopher Wallace Email address: cwallace195@live.co.uk

Refereed Paper.

Accepted 24 September 2020

https://doi.org/10.1038/s41415-021-3015-y specialist for consultation and treatment planning. Remote monitoring occurs when a general dental practitioner (GDP) or specialist gathers periodic photos and information from the patient to monitor treatment progress or development of a condition, while video conferencing involves real-time interaction via video calls. ${ }^{2}$

As a result of the COVID-19 pandemic, teledentistry has been utilised more frequently due to social distancing regulations. ${ }^{3}$ Minimising the number of face-to-face appointments and reducing the volume of people in clinical areas and inappropriate attendances is key to reducing the risk of viral transmission. Remote monitoring and management of dental conditions has never been more important to protect patients, the public and the dental team. ${ }^{4}$ Teledentistry has previously been evaluated for orthodontic new patients, but not within paediatric dentistry. ${ }^{5}$
In the orthodontic study, it was concluded that teledentistry was a valid system for appropriately screening new patient referrals and reducing the inappropriate referral rate, but it had no impact on likelihood of appointment attendance. Furthermore, they acknowledged that there is a risk that a patient who is not accepted on the teledentistry system would ultimately benefit from a full clinical examination, with one in six patients deemed inappropriate via teledentistry referral being considered appropriate when examined clinically. ${ }^{5}$

Within the Newcastle upon Tyne Hospital NHS Foundation Trust, paediatric dentistry is located within the dental directorate. The Child Dental Health Department within Newcastle Dental Hospital provides a regional paediatric dentistry service for North East England, which has an estimated population of 502,000 children under the age of $16 .{ }^{6}$ Prior 
to the COVID-19 pandemic, 80-100 new patients per week were assessed via face-toface dental assessments. On average, patients were usually in the department for two hours for their first visit. Following the advice from NHS England and the Trust, routine dental services were suspended from 23 March $2020^{7}$ and urgent care was provided via a telephone triaging system. ${ }^{8}$ From mid-May 2020, new patient appointments within the Child Dental Health Department were provided via telephone consultations. This represented a unique opportunity to assess the advantages and disadvantages of telephone consultations for new patients within a specialist paediatric dentistry service.

This service evaluation aimed to assess the potential roles of teledentistry on a paediatric dentistry new patient clinic, and evaluate patient pathways and the advantages and disadvantages of using this service method. In addition, we aimed to determine if teledentistry could have a role in the 'post-COVID-19' world, in line with the digitally enabled care described in the NHS Long Term Plan. ${ }^{9}$

\section{Method}

Data were collected from remote 'teledentistry' new patient clinics in the paediatric dentistry department at Newcastle Dental Hospital. All patients had been referred before 20 March via referral letters but were not seen before the COVID-19 pandemic. Data were collected for all patients telephoned for a one-month period (13 May-12 June 2020) using a set pro forma completed by clinicians after each consultation. Information collected included age, referral source, medical and social history, interpreter requirement, presumed dental diagnosis, outcome and priority rating. Data were collected from clinical notes and recorded using a Microsoft Excel spreadsheet that was safely stored on a Trust-approved encrypted computer. Data were anonymised and the project was registered with the Newcastle upon Tyne Hospital NHS Foundation Trust as a service evaluation. All referrals were graded against the tiered system found in Commissioning standard for dental specialties - paediatric dentistry to assess what level of expertise was required to successfully manage each patient. ${ }^{10}$

The department operates a partial booking system for new patient appointments and therefore all parents/guardians contacted the booking team to book a date and time for their

\section{Fig. 1 Referral source}

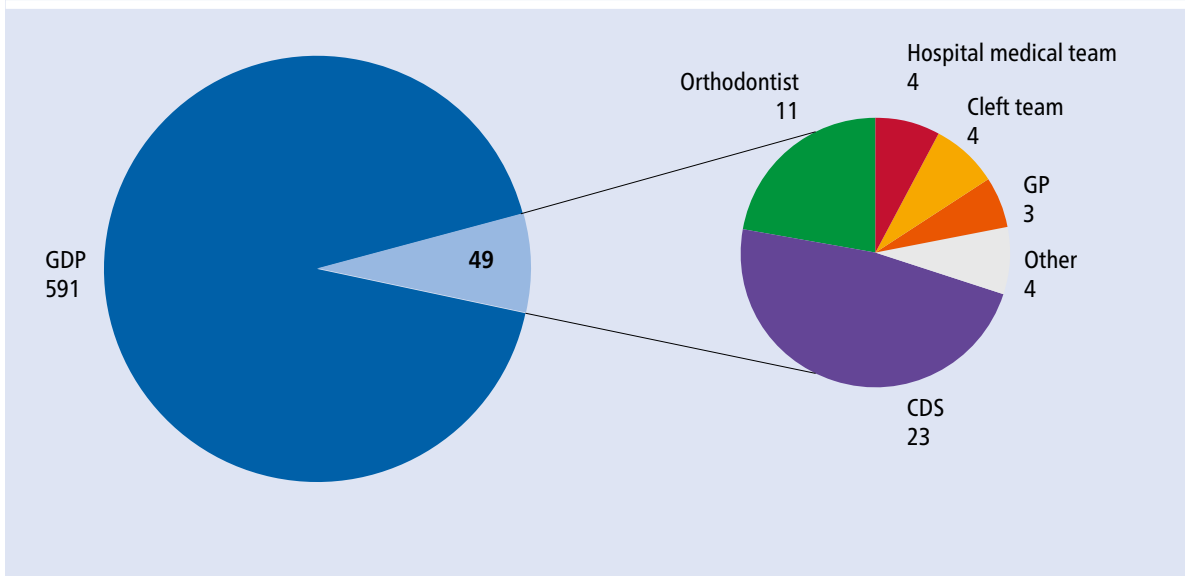

Fig. 2 Tier of referrals

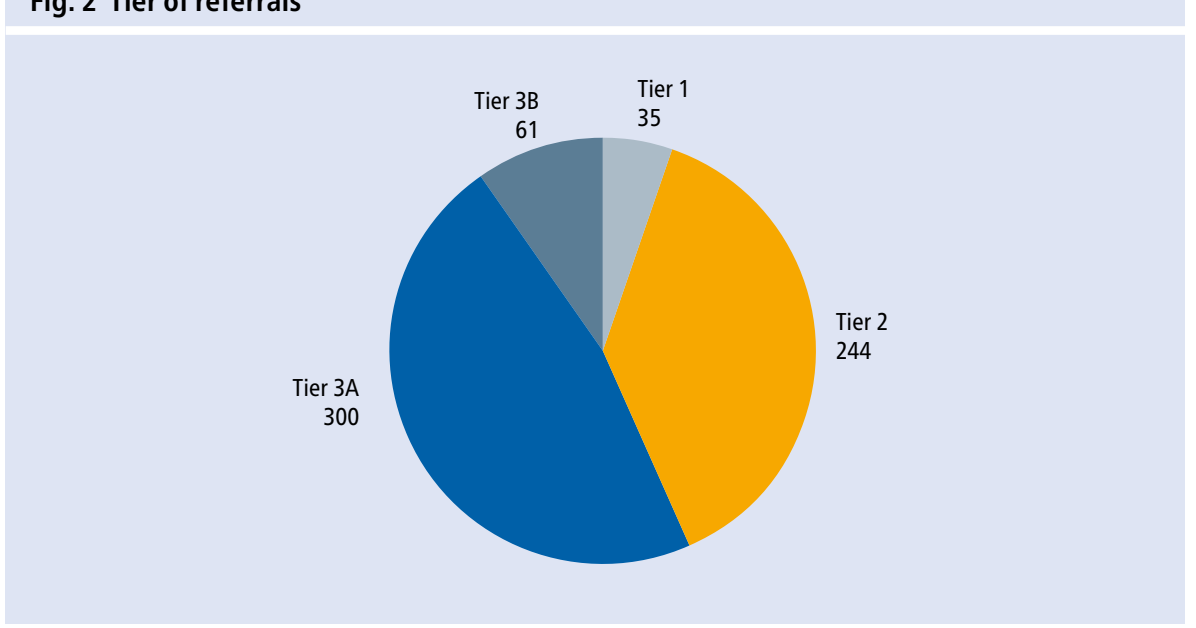

telephone appointment. For those patients who required interpreter services, they were contacted via a three-way teleconference call using the 'BigWord' telephone interpreting service. If photos were deemed useful, parents were asked to e-mail photos to a secure e-mail address for review by the clinical team. During the call, patients were triaged on the basis of their clinical need and allocated to an appropriate patient pathway.

Failure to respond to telephone calls was also documented. Where patients could not be reached on the contact numbers provided, background checks were completed to find any alternative ways of contacting the parents or guardians, before sending an 'await contact' letter. All patients deemed suitable for discharge had further background checks completed to ensure they did not have any flagged safeguarding concerns before discharge. Letters were sent to all patients, their dentists, and (if appropriate) their doctors and social workers to inform them of the outcome following the telephone consultation.

\section{Results}

In total, 653 patient appointments were booked during the audit period. Of these 653 appointments, 518 (79\%) had successful contacts and 135 (21\%) had unsuccessful initial contacts. Of these 135 unsuccessful initial contacts within the audit period, 13 (10\%) re-contacted the department and had a telephone consultation, while 122 failed to do so. However, for those who were not contacted, the 'await contact' letter took time to generate and so not all of these patients would have received this before the end of the assessment period. Therefore, the estimated percentage of unsuccessful contacts who do get back in touch is likely to be higher than the $10 \%$ estimated by this service evaluation.

With the 13 duplicates removed, 640 patients in total had booked appointments. The majority of these referrals were from primary care GDPs, as illustrated by Figure 1, and referral tiers are shown in Figure 2. Twentyfive patients $(4 \%)$ required an interpreter, of whom 18 (72\%) had successful contacts. 
Table 1 Dental diagnosis of all contacted patients

\begin{tabular}{|c|c|}
\hline Diagnosis & Number \\
\hline Caries - primary dentition & 175 \\
\hline $\mathrm{MIH}$ & 73 \\
\hline Caries - permanent dentition & 62 \\
\hline Enamel/dentine defect - not MIH (aesthetic management) & 21 \\
\hline Trauma - permanent dentition & 19 \\
\hline Hypodontia & 14 \\
\hline Soft tissue lesions & 14 \\
\hline Trauma - primary dentition & 13 \\
\hline Caries - both & 12 \\
\hline Orthodontic extractions & 12 \\
\hline Infraocclusion & 11 \\
\hline Supernumerary - erupted & 10 \\
\hline Supernumerary - unerupted & 7 \\
\hline Non-vital tooth & 7 \\
\hline Retained primary teeth & 7 \\
\hline Enamel/dentine defect - not MIH (non-aesthetic management) & 6 \\
\hline Other orthodontic needs (for example, crowding/altered tooth position/eruption) & 6 \\
\hline Periodontal & 6 \\
\hline Hypomineralised primary molars & 5 \\
\hline TMJD & 5 \\
\hline Primary failure of eruption & 5 \\
\hline Delayed/altered dental development & 5 \\
\hline NCTSL & 4 \\
\hline Diagnosis unclear from referral and history & 4 \\
\hline Impaction & 3 \\
\hline Endodontic troubleshooting & 3 \\
\hline High frenal attachments/frenal issues & 3 \\
\hline Hard tissue anomaly & 2 \\
\hline No pathology & 2 \\
\hline Bruxism & 1 \\
\hline Neonatal tooth & 1 \\
\hline Total & 518 \\
\hline
\end{tabular}

The most common reasons for referral were caries in the primary dentition, molarincisor hypomineralisation (MIH) and caries in the permanent dentition (Table 1). Following telephone consultation, $3 \%$ of patients required urgent assessment and 15\% priority face-to-face assessment, while 59\% required a routine face-to face appointment and $21 \%$ were discharged (Fig. 3). Other less common outcomes included telephone review, video consultation, onward referral to other specialties and arrangements for 'piggyback' general anaesthetic in liaison with other surgically planned general anaesthetics.

Overall, 114 patients were discharged back to their dentist because treatment was no longer required, or they required re-referral at a later stage. Common reasons for this discharge (Table 2) included asymptomatic MIH cases referred before the ideal window for loss of first permanent molars, ${ }^{11}$ hypodontia patients who had been referred too soon for orthodontic treatment and patients requiring aesthetic treatment or orthodontic extractions, which were not currently being offered due to COVID-19 restrictions. Other discharge reasons included cases where children had already received treatment successfully under local anaesthetic by their general dentist, cases which were suitable for tier 1 treatment in primary care and those with conditions which had resolved since the date of referral, such as temporomandibular joint dysfunction (TMJD) or resolved concerns about dental development.

\section{Discussion}

This was a pragmatic review conducted to evaluate a new service offered by our department in response to the global pandemic situation. Nevertheless, there are several valuable learning points that arose during the course of our evaluation.

Purely on a numbers basis, this method allowed us to validate our waiting list. Previously, all 640 patients would have been offered a face-to-face appointment on a consultant-led clinic. By implementing a telephone consultation as the first point of contact with our team, we were able to reduce the number of face-to-face appointments required by over a third. Our 'failed to contact' rate during the period of the service evaluation was $21 \%$; this is comparable to our 'was not brought' rate to our new patient clinics of $18 \%$ during the same period in the previous year.

\section{Key points for GDPs}

There have been some key points highlighted in this study, which are important for our colleagues in general dental practice to be aware of.

Firstly, the importance of enclosing up-todate demographic details within the referral. In the sample, $21 \%$ of patients' parents did not answer their phones and were uncontactable. This may be because addresses or phone numbers had changed since initial referral or due to the fact that calls from our hospital are from 'withheld' numbers, which may have led to patients being reluctant to answer these calls. Additionally, the quality of our telephone consultation was significantly influenced by the quality of the referral. Referrals where radiographs and/or clinical photographs were enclosed were more likely to have a definitive outcome to the telephone consultation. 


\section{Fig. 3 Outcomes of telephone assessment}

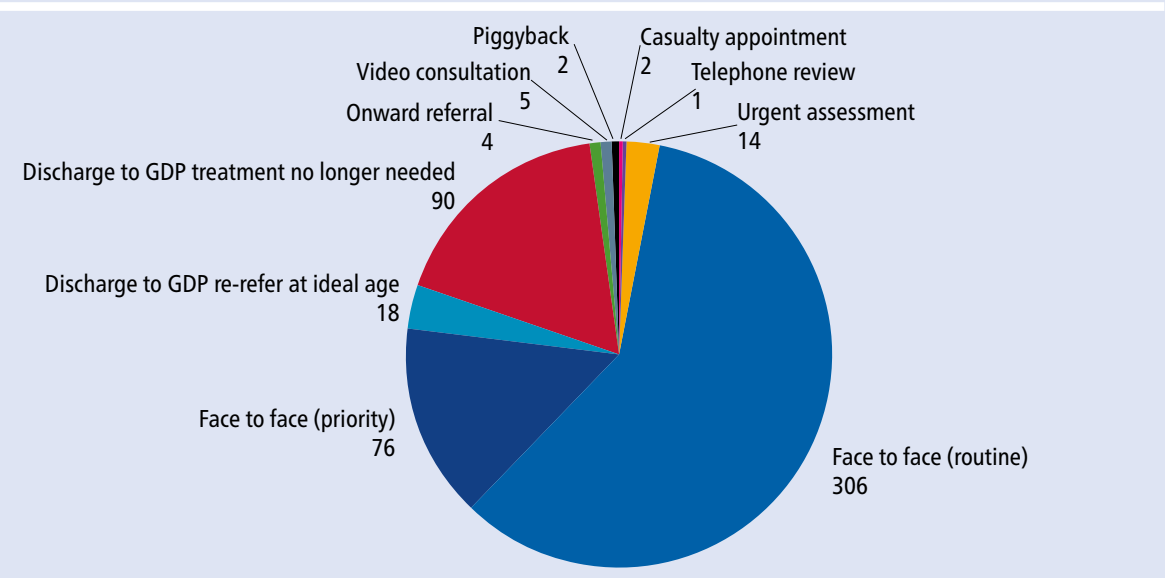

Table 2 Dental diagnoses of discharged contacted patients

\begin{tabular}{|c|c|}
\hline Diagnosis & Number \\
\hline $\mathrm{MIH}$ & 20 \\
\hline Enamel/dentine defect - not MIH (aesthetic management) & 19 \\
\hline Orthodontic extractions & 12 \\
\hline Caries - primary dentition & 8 \\
\hline Caries - permanent dentition & 7 \\
\hline Hypodontia & 6 \\
\hline Other orthodontic needs (for example, crowding/altered tooth position/eruption) & 4 \\
\hline Trauma - primary dentition & 4 \\
\hline Diagnosis unclear from referral and history & 3 \\
\hline High frenal attachments/frenal issues & 3 \\
\hline Trauma - permanent dentition & 3 \\
\hline Delayed/altered dental development & 2 \\
\hline No pathology & 2 \\
\hline Periodontal & 2 \\
\hline Primary failure of eruption & 2 \\
\hline Retained primary teeth & 2 \\
\hline Soft tissue lesions & 2 \\
\hline Supernumerary - erupted & 2 \\
\hline Supernumerary - unerupted & 2 \\
\hline TMJD & 2 \\
\hline Bruxism & 1 \\
\hline Enamel/dentine defect - not MIH (non-aesthetic management) & 1 \\
\hline Endodontic troubleshooting & 1 \\
\hline Hypomineralised primary molars & 1 \\
\hline Impaction & 1 \\
\hline Infraocclusion & 1 \\
\hline Neonatal tooth & 1 \\
\hline Total & 114 \\
\hline
\end{tabular}

Secondly, timing of referral is important for several conditions. Twenty of the patients who were discharged back to their dentist had asymptomatic first permanent molars of poor prognosis that had been referred 'too early'. Ideally in this situation, children should be referred at 'dental age' of 8-8.5. ${ }^{11}$ We were also able to discharge children who had been referred due to developmentally absent permanent teeth but were still in the early mixed dentition. Once it is identified that permanent teeth are absent, it is best to refer these patients when they are close to an established permanent dentition, unless there are concerns regarding the position of the maxillary permanent canines.

Thirdly, it is worth liaising with your provider of orthodontic services before completing any orthodontic extractions at present. With the COVID-19 restrictions, it may be that the orthodontist is prioritising continuation of existing cases rather than starting new cases. If orthodontic extractions are not timed with the commencement of treatment, this can mean space for correction of the malocclusion is lost, or if it is a long time since the extractions were prescribed, the treatment plan may have altered due to facial growth.

\section{Applications for specialist paediatric dentistry units}

The following applications of teledentistry should be considered by paediatric dentists if developing a similar service within their unit.

\section{Preventative advice}

Delivering preventative advice via telephone would allow patients to complete the preventative phase of their treatment plans before initial assessment. Diet diaries can be e-mailed or posted in advance and patients can be signposted to online oral hygiene resources. Older adolescent patients can be signposted to alcohol or smoking support services, if appropriate. Remote prescription of highstrength sodium fluoride toothpaste could be completed where required, in line with national guidelines such as the Delivering better oral health toolkit. ${ }^{12}$ By reinforcing preventative advice at the initial telephone appointment, this acts as a useful adjunct to help transition the patient from an active to inactive oral disease state. This can be completed by both primary and secondary care, especially in a climate where face-to-face contact is restricted. Many dental nurses are trained in oral health education and could therefore also support clinicians with teledentistry prevention appointments. 
Non-carious tooth surface loss (NCTSL) corresponds especially well with initial teleconsultation given that, for most cases, NCTSL will need to be stable before commencing any definitive restorative treatment. ${ }^{13}$ History taking can quickly evaluate whether NCTSL is due to extrinsic or intrinsic acids, attrition or abrasion, or a combination of these factors. Should a patient indicate problems with intrinsic acids through gastro-oesophageal reflux disease, chronic vomiting or an eating disorder (such as bulimia nervosa), then they can be signposted to their general medical practitioner (GMP) for assessment before attending their dental appointment. ${ }^{13}$ Desensitising toothpaste advice can also be given remotely should sensitivity be experienced.

\section{Assessment of dental development}

Some patients may be referred for delayed eruption or exfoliation of teeth. However, during the waiting period from referral to initial appointment, these teeth may naturally erupt or exfoliate. Therefore, an initial remote video call or photographic assessment may help establish if further specialist assessment is necessary or whether the patient can be reassured and discharged. Teledentistry could also be useful for paediatric dentists or orthodontists who are awaiting exfoliation of primary teeth before commencing definitive treatment. Other developmental conditions which could be monitored by teledentistry include infraoccluded teeth, neonatal/natal teeth and eruption cysts.

\section{Oral medicine conditions}

In oral medicine cases, for paediatric patients with obviously benign soft tissue conditions such as mucoceles, fibroepithelial polyps or eruption cysts, a teledentistry review could offer parents quick reassurance and provide the clinician with confidence that those children can be safely placed on a routine waiting list. However, for lesions with worrying features, teledentistry would allow these patients to be prioritised for urgent assessment. A previous feasibility study of teledentistry 'store and forward' oral medicine referrals in Northern Ireland assessed 37 adult patients and showed that this was an acceptable method of information transfer for both patients and clinicians, with all technical aspects of the process proving satisfactory. ${ }^{14}$

A further advantage of teledentistry is that it allows clinicians to arrange relevant further investigations immediately upon receipt of referral. For example, for patients with recurrent aphthous stomatitis, they could have blood tests completed by their GMP and complete a diet diary in preparation for their initial assessment. ${ }^{15}$ Remote prescribing may be appropriate for certain groups of new patients to reduce discomfort in the interim while waiting for their initial assessment. For example, benzydamine hydrochloride (Difflam) mouthwash may be helpful in those with post-chemoradiotherapy mucositis or those with recurrent aphthous stomatitis or erosive conditions. ${ }^{15}$

\section{Temporomandibular joint disorder}

A relatively comprehensive TMJD history and examination can take place via teledentistry. Any 'red flags' can be established ${ }^{16}$ and remote examination for myofascial pain, arthralgia, altered jaw movement and cranial nerve abnormalities is generally possible via video consultation. Basic TMJD advice such as information regarding analgesics, stress management, topical compresses, avoidance of jaw habits and physiotherapy can all take place remotely. ${ }^{17}$

\section{Patients with additional social needs}

For those more vulnerable patients who are currently under the care of social services or receiving additional support, a teledentistry review can help a clinician establish the nature of a child's social status in advance of their appointment. This can help them liaise with other services as necessary to ensure the family receives extra support as needed to enable them to attend appointments. An initial telephone review may also be helpful to build rapport and reduce anxiety in those patients or parents with dental or social phobias.

Teledentistry can also improve accessibility to care for those who do not speak English. At Newcastle Dental Hospital, three-way teleconference calls with telephone interpreters have been invaluable. Teledentistry can also be used to help bridge the rural-urban divide and improve accessibility to care for more isolated communities. $^{18}$

\section{Implications for our patients and their parents/guardians}

From a patient or parental point of view, one of the biggest advantages of teledentistry is that it reduces the need for face-to-face attendance, thereby reducing inconvenience and the costs associated with travel and time off work and school. ${ }^{19}$ In this study, over the course of one month, there was in the region of 190 hours of school and work time saved, and therefore also the financial implications which come with this. Furthermore, given the current COVID-19 pandemic, parents may prefer not to attend hospital appointments and teledentistry therefore offers a suitable alternative for those who do not require urgent face-to-face assessment. ${ }^{3}$ Teledentistry may also be preferred by those who are housebound, the elderly or those who struggle to leave the house either through physical or mental disability (for example, severe anxiety or agoraphobia). ${ }^{14}$ An initial telephone call may help break down some of the perceived barriers between the patient and clinician, and a friendly call may help patients and their parents feel less apprehensive before their visit. Additionally, by discussing potential diagnoses or treatment options in advance of attending for initial assessment, this may give patients and parents more time to think and come up with any questions they may have, thereby improving the consent process. At Newcastle Dental Hospital, this has been especially useful for discussions surrounding the risks, benefits and process of inhalation sedation or general anaesthesia. Clinicians have also been able to advise parents of alternative options where appropriate; for example, the Hall technique, silver diamine fluoride and conventional restorations for management of primary caries.

However, some patients may not be able to access teledentistry due to the cost associated with owning a mobile phone or computer. Furthermore, teledentistry may be unsuitable for certain patient groups, such as those who are hard of hearing or who require additional communication aids. If remote interpreting services were not available, this would also be a significant barrier to those who do not speak English. Background distractions during phone calls may also interfere with how well messages are received and the lack of face-toface interaction may be off-putting for some patients where depersonalisation can act as a significant barrier to communication. ${ }^{20}$

\section{Medico-legal implications}

The biggest concern surrounding teledentistry from a clinical point of view is the risk of misdiagnosis and subsequent mismanagement of a patient, which could lead to patient harm or medico-legal issues. ${ }^{19,20}$ Accuracy of diagnosis can be influenced by the quality of images received, which may be dependent on the cooperation of the patient and quality 
of IT devices owned by both the referrer and receiving dentist. Dental Protection, a UK-based dental indemnity provider, advises that dentists should always recommend the most appropriate route for patient care in line with their local public health and government guidelines. ${ }^{21}$ Dentists should only assess a patient remotely if they feel they can complete an adequate assessment and they should readily direct patients to local dental services when required. Remote prescribing should only be considered when clinically justified and face-to-face consultation is not possible. ${ }^{21}$

\section{Conclusions}

Teledentistry is an emerging field and has multiple potential applications in the field of paediatric dentistry. Appropriate use of a teledentistry system can help improve patient journeys, reduce waiting lists and reduce unnecessary face-to-face attendances, which is especially important to ensure patient safety during the current COVID-19 pandemic. However, care must be taken to ensure a clinician is confident in their diagnosis before discharging or delaying management of a patient. Medico-legal issues, financial implications and data protection also need to be fully considered before implementing such a service. Many of the uses of teledentistry highlighted in this service evaluation are applicable for both primary and secondary care. As COVID-19 restrictions ease, teledentistry can provide a useful adjunct in the areas discussed.

On a local level, the Child Dental Health Department at Newcastle Dental Hospital has adapted the referral triaging protocol to include telephone or video consultations. This has allowed for prioritisation of patients and rejection of inappropriate referrals without lengthy face-to-face consultations. It is likely that as initial contact via telephone continues, the system will be developed further and potentially integrated as a routine method for new patient contact. In addition, the referral criteria and associated paperwork are being revised in light of the information obtained from this review. Future areas to explore may include: a collaborative audit with other units to assess similarities and differences between services and geography; undertaking a similar review within the department to assess impact on referrers' practice; and collection of additional data in further work such as distance to hospital and travel time to further quantify the benefits to patients.

\section{Conflict of interest}

None declared.

\section{Acknowledgements}

Thanks to all members of staff on the Child Dental Health Clinic at Newcastle who were involved in telephone consultations and helped with our data collection by completing audit sheets.

\section{References}

1. Chen J, Hobdell M, Dunn K, Johnston K, Zhang J. Teledentistry and its use in dental education. J Am Dent Assoc 2003; 134: 342-346.

2. Jampani N, Nutalapati R, Dontula B S K, Boyapati R. Applications of Teledentistry: A literature review and update. J Int Soc Prev Community Dent 2011; 1: 37-44.

3. World Health Organisation. Coronavirus disease (COVID19) advice for the public. 2020. Available at https://www. who.int/emergencies/diseases/novel-coronavirus-2019/ advice-for-public (accessed June 2020).

4. Giudice A, Barone S, Muraca D et al. Can Teledentistry improve the monitoring of patients during the COVID-19 dissemination? A descriptive pilot study. L. Int J Environ Res Public Health 2020; 17: 3399-4007.

5. Mandall N A, O'Brien K D, Brady J, Worthington H V Harvey L. Teledentistry for screening new patient orthodontic referrals. Part 1. A randomised controlled trial. Br Dent J 2005; 199: 659-662.

6. Office for National Statistics. Census Output area population estimates - North East, England. 2019. Available online at https://www.ons. gov.uk/peoplepopulationandcommunity/ populationandmigration/populationestimates/ datasets/censusoutputareaestimatesinthenortheast regionofengland (accessed July 2020)
7. Faculty of General Dental Practice (UK). COVID-19: routine dental care ends throughout the UK. 2020. Available at https://www.fgdp.org.uk/news/covid-19-routine-dentalcare-ends-throughout-uk (accessed July 2020).

8. Simpson S, Sumner O, Holliday R et al. Paediatric Dentistry and the coronavirus (COVID-19) response in the North East of England and North Cumbria. 2020. Available online at https://doi. org/10.1101/2020.06.02.20114967 (accessed July 2020)

9. NHS. The NHS Long Term Plan. 2019. Available online at https://www.longtermplan.nhs.uk/publication/nhslong-term-plan/ (accessed August 2020).

10. NHS England. Commissioning Standard for Dental Specialties - Paediatric Dentistry. 2018. Available at https://www.england.nhs.uk/wp-content/ uploads/2018/04/commissioning-standard-for-dentalspecialties-paediatric-dentristry.pdf (accessed June 2020).

11. Cobourne M T, Williams A, Harrison M. A guideline for the extraction of first permanent molars in children. 2014. Available online at https://www.rcseng.ac.uk/ dental-faculties/fds/publications-guidelines/clinicalguidelines/ (accessed June 2020).

12. Public Health England. Delivering better oral health: an evidence-based toolkit for prevention. Third edition. 2017. Available at https://assets.publishing.service.gov. uk/government/uploads/system/uploads/attachment data/file/605266/Delivering_better_oral_health.pdf (accessed June 2020).

13. Haemmings K, Truman A, Chauhan R. Tooth wear guidelines for the BSRD Part 1: Aetiology, Diagnosis and Prevention. Dent Update 2018; 45: 3-10.

14. Bradley M, Black P, Noble S, Thompson R, Lamey PJ. Application of Teledentistry in Oral Medicine in a Community Dental Service, Northern Ireland. Br Dent J 2010; 209: 399-404.

15. Felix D H, Luker J, Scully C. Oral Medicine: 1. Ulcers: Aphthous and other common ulcers. Dent Update 2012; 39: 513-519.

16. Hegarty A M, Zakrzewska J M. Differential Diagnosis for Orofacial Pain including sinusitis, TMD, Trigeminal neuralgia. Dent Update 2011; 38: 396-406.

17. Yule P L, Durham J, Wassell R W. Pain Part 6: Temporomandibular Disorders. Dent Update 2016; 43: $39-48$.

18. Nuttall N M, Steed M S, Donachie M A. Referral for Secondary restorative dental care in rural and urban areas of Scotland: findings from the Highlands and Islands Teledentistry Project. Br Dent J 2002; 192: 224-228.

19. Mandall N A, Quereshi U, Harvey L. Teledentistry for screening new patient orthodontic referrals. Part 2: A GDP perception of the referral system. Br Dent J 2005; 199: 727-729.

20. Patel R N, Antonarakis G S. Factors influencing the adoption and implementation of teledentistry in the UK, with a focus on orthodontics. Community Dent Oral Epidemiol 2013; 41: 424-431.

21. Dental Protection. COVID-19 and remote consultations - how we can help. 2020. Available at https://www. dentalprotection.org/uk/articles/covid-19-and-remoteconsultations-how-we-can-help (accessed June 2020). 